Génét. Sél. Evol., 1986, 18 (2), 225-236

\title{
Energie métabolisable et digestibilité azotée chez les poulets en croissance : influence du génotype, de l'âge et du régime ${ }^{(1)}$
}

\author{
A.S. HASSAN et P. DELPECH \\ Institut National Agronomique Paris-Grignon \\ Centre de Grignon, F 78850 Thiverval-Grignon
}

\begin{abstract}
Résumé
Des mesures de l'énergie métabolisable et de la digestibilité azotée sont réalisées avec des poulets possédant 4 génotypes différents : 2 possèdent le gène de nanisme $d w$ mais ont des vitesses de croissance différentes ( $J V 15$ et $J A$ 57), un autre est un croisement commercial pour la ponte d'œufs teintés (ISA Brown), le dernier est une souche du Fayoumi, race égyptienne. Les mesures portent sur 3 régimes formulés pour être isoénergétiques, différant par leurs taux azotés. Elles sont réalisées entre 30 et 35 jours d'une part, 55 et 60 jours d'autre part. Les ISA Brown métabolisent moins bien l'énergie que les autres. La valeur de l'énergie métabolisable corrigée pour une rétention azotée nulle augmente avec l'âge. Ces variations pourraient s'expliquer par des variations de la digestibilité des lipides.

La digestibilité azotée est inférieure chez les ISA Brown. Elle diminue avec l'âge pour tous les génotypes sauf pour les Fayoumi. Il existe une corrélation élevée durant la première période entre l'énergie métabolisable et le rapport :
\end{abstract}

matière sèche totale des fientes excrétées

matière sèche totale des aliments ingérés

Mots clés: Poule, génotype, âge, énergie métabolisable, digestibilité azotée.

\begin{abstract}
Summary
Metabolisable energy value and protein digestibility in chickens : influence of genotype, age and diet
\end{abstract}

Metabolisable energy value and protein digestibility of the feed have been measured on 4 genetically different groups of chickens. Two have the $d w$ dwarfism gene but differ in growth rate (JV 15 and $J A 57)$, the third is a commercial cross for coloured egg production (ISA Brown), the fourth is a strain within the Egyptian breed Fayoumi. Measurements have been made with 3 diets designed to be isoenergetic but with 3 levels of crude protein

(1) Ce travail fait partie de la thèse de A.S. Hassan à l'Institut National Polytechnique de Toulouse. 
content, between 30 and 35 days of age and between 55 and 60 days of age. The ISA Brown group has a poorer energy-metabolism than the others. The value of metabolisable energy adjusted to a zero nitrogen retention increases with age. Such variations could be explained by lipid-digestibility variations. Crude protein digestibility is lower for ISA Brown and decreases with age for all groups but Fayoumi. Correlation is high between metabolisable energy and the ratio :

excreta total dry matter

ingesta total dry matter

Key words : Chicken, genotype, age, metabolisable energy, crude protein digestibility.

\section{Introduction}

La comparaison de mesures de l'énergie métabolisable (EM) entre oiseaux ayant des patrimoines héréditaires différents a déjà fait l'objet de 2 revues. Miller (1974) estime que, lorsqu'elles existent, les différences entre génotypes sont de faible amplitude, inférieure à 3 p. 100. LECLERcQ (1980) considère que ces différences entre génotypes proviennent d'erreurs de mesure liées aux méthodes d'estimation des bilans énergétique et azoté; l'emploi de traceur (le plus souvent oxyde de chrome) donne des résultats moins précis que la collecte totale. Les divergences peuvent aussi provenir du coefficient de correction utilisé pour corriger l'EM ou une rétention azotée nulle.

Toutefois il faut remarquer que les souches Rhode-Island Red paraissent métaboliser moins bien l'énergie que les autres (BEGIN, 1967 et 1969; FosTER, 1968). Pour certains, les souches chair utilisent l'énergie plus faiblement que les Leghorn (Sibbald \& Slinger, 1963 ; Slinger et al., 1964) tandis que d'autres constatent le contraire, même si les différences ne sont pas significatives (BEGIN, 1967 et 1969). Pour Proudman et al. (1970), ce sont les animaux à croissance rapide issus d'une sélection divergente sur la vitesse de croissance, qui donnent les valeurs d'EM les plus élevées. En outre, chez les poules pondeuses, FosTER (1968) constate que des différences significatives existent entre des individus d'une même souche ou croisement. Une nouvelle évaluation des différences entre oiseaux possédant des patrimoines génétiques différents s'avère utile.

L'âge auquel a été effectuée la mesure et la composition des régimes peuvent également expliquer ces contradictions. En effet, si on se réfère aux résultats obtenus à l'I.N.R.A. (Anonyme, 1984), la valeur de l'énergie métabolisable des aliments est plus élevée, ou plus faible quand elle est mesurée respectivement sur des coqs ou des poussins. Même dans le cas des lipides, Lessire et al. (1982) ont montré que les variations de digestibilité des lipides entre 2 et 6 semaines ne pouvaient expliquer entièrement celle de l'EM, quel qu'en soit le mode d'expression. Cependant, 2 études montrent que, pour des régimes mixtes, il existe une augmentation de l'EM entre 20 et 35 jours, suivie d'un plateau, plutôt que d'une chute comme l'indique Zelenka (1968). L'étude des valeurs observées par SoRENSEN et al. (1983) semble indiquer que ce plateau est atteint plus rapidement pour des animaux sélectionnés pour utiliser les régimes à bas taux azoté. Une comparaison avant et après 5 semaines devrait permettre de vérifier l'influence de l'âge sur les variations éventuelles d'origine génétique.

Enfin, les résultats obtenus en faisant varier le taux azoté du régime s'avèrent également contradictoires. Seuls des taux azotés élevés permettent de mettre en évi- 
dence des différences de la valeur énergétique des graisses (Sibbald et al., 1961). En revanche, Fonolla et al. (1981) ne constatent pas d'effet du taux protéique quand celui-ci varie de 19 à 24 p. 100. Mais il n'existe pas d'études au cours desquelles la concentration azotée soit diminuée nettement en-dessous du besoin.

En ce qui concerne la digestibilité azotée, il n'existe pas d'études relatives à l'influence du génotype. La digestibilité azotée paraît plus élevée chez les jeunes d'après HẢKANSSON \& ERIKSSON (1974) et FonOlla et al. (1981). Elle paraît influencée défavorablement par la baisse du taux azoté et la présence d'urée (Fonolla et al., 1981). Il nous a donc semblé utile d'associer la mesure de la digestibilité azotée à celle de l'EM et d'examiner les relations éventuelles entre elles.

\section{Matériels et méthodes}

\section{A. Animaux}

Les poulets utilisés proviennent de 4 souches ou croisements différents. Les $J V 15$ et $J A 57$ sont des croisements entre 2 lignées nanifiées possédant le gène $d w$. Ils fournissent les mères des poussins commerciaux de l'Institut de Sélection Animale (ISA). Les ISA Brown résultent du croisement entre 4 lignées de Rhode-Island Red : les femelles constituent les troupeaux de pondeuses commercialisées par l'ISA. Les Fayoumi proviennent d'une souche de cette race égyptienne sélectionnée à l'Université du Caire. Tous les sujets sont de sexe mâle.

\section{B. Mode d'élevage}

Les poussins mâles sont élevés en cages collectives jusqu'à l'âge de 10 jours, puis placés en cages individuelles. Les Fayoumi ont été introduits dans notre élevage seulement à l'âge de 27 jours. Les animaux sont maintenus à une température d'ambiance à peu près constante. Elle est fixée à $24^{\circ} \mathrm{C}$ à partir de 21 jours. L'éclairement est limité à 14 heures à partir du $5^{\circ}$ jour. L'eau et les régimes sont distribués individuellement, à volonté.

\section{Lots et régimes}

Dans chaque génotype, 52 animaux sont choisis parmi 80 en fonction de leur poids à 10 jours, de manière à éliminer les plus légers et les plus lourds. Ils sont répartis en 2 lots de 26 sujets ayant le même poids moyen. Les régimes alimentaires diffèrent par leurs taux azotés, respectivement élevé et bas et sont distribués en farine. Trois mélanges ont été calculés de manière à réaliser des régimes isocaloriques dont les taux azotés s'élèvent respectivement à 20,18 et 14,3 p. 100 de protéines brutes. La composition en est décrite dans le tableau 1. Les apports calculés en acides aminés sont équilibrés par rapport à la concentration azotée. Ainsi pour chaque lot la succession des régimes correspond au mélange à 20 p. 100 suivi de celui à 18 p. 100 ou à la succession des régimes à 18 p. 100 suivi de celui à 14,3 p. 100 ; le changement d'aliment s'effectue à l'âge de 35 jours (tabl. 2). 


\section{TABLEAU 1}

Composition des régimes en p. 100.

Composition of the diets

\begin{tabular}{|c|c|c|c|}
\hline Aliments & 1 & 2 & 3 \\
\hline Blé . & 13,1 & 30 & 26,0 \\
\hline Maïs $\ldots \ldots \ldots \ldots \ldots$ & 47,2 & 37,84 & 40,7 \\
\hline Tourteau de soja $\ldots \ldots \ldots \ldots \ldots \ldots$ & 29,4 & 18,40 & 13,8 \\
\hline Tourteau de tournesol $\ldots \ldots \ldots \ldots \ldots$ & - & 4,10 & 一 \\
\hline Farine de viande $\ldots \ldots \ldots \ldots \ldots \ldots$ & 2,0 & 1,90 & 1,2 \\
\hline 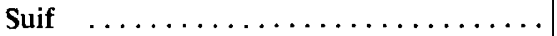 & 5,0 & 4,50 & 2,2 \\
\hline Manioc $\ldots \ldots \ldots \ldots \ldots \ldots \ldots \ldots \ldots$ & - & - & 13,0 \\
\hline Phosphate bicalcique $\ldots \ldots \ldots \ldots \ldots$ & 1,0 & 1,0 & 1,1 \\
\hline Carbonate de calcium & 1,22 & 1,26 & 1,0 \\
\hline Composé minéral et vitaminique $\ldots \ldots$ & 1,0 & 1,0 & 1,0 \\
\hline DL Méthionine $\ldots \ldots \ldots \ldots \ldots \ldots$ & 0,08 & - & - \\
\hline Composants calculés $\ldots \ldots \ldots \ldots \ldots$ & & & \\
\hline Protéines brutes $\ldots \ldots \ldots \ldots \ldots \ldots \ldots$ & 20,9 & 18,0 & 14,3 \\
\hline Lysine $\quad \ldots \ldots \ldots \ldots \ldots \ldots \ldots \ldots$ & 1,10 & 0,84 & 0,65 \\
\hline Méthionine + Cystine $\ldots \ldots \ldots \ldots \ldots$ & 0,77 & 0,63 & 0,50 \\
\hline Energie métabolisable coq $\ldots \ldots \ldots \ldots$ & 3000 & 3000 & 2990 \\
\hline
\end{tabular}

TABleau 2

Séquences d'alimentation des lots.

Diets succession.

\begin{tabular}{|c|c|c|}
\hline \multirow{2}{*}{ Période } & \multicolumn{2}{|c|}{ Taux azoté en p. 100} \\
\hline & élevé & bas \\
\hline $10 \mathrm{j}$ à $35 \mathrm{j}$ & 20,9 & 18,0 \\
\hline $36 \mathrm{j}$ à $60 \mathrm{j}$ & 18,0 & 14,3 \\
\hline
\end{tabular}

\section{Enregistrements}

La croissance pondérale et la consommation sont contrôlées à 10,20, 30 et 35 jours pour la première période ; à 45,55 et 60 jours pour la deuxième période. Les bilans sont réalisés entre le $30^{\mathrm{e}}$ et le $35^{\mathrm{e}}$ jour et entre le $55^{\mathrm{e}}$ et le $60^{\circ}$ jour. Les oiseaux sont d'abord soumis à un jeûne de $24 \mathrm{~h}$; ils reçoivent ensuite leurs régimes habituels pendant 3 jours. Le $5^{\mathrm{e}}$ jour correspond également à une période de jeûne. Cette séquence est semblable à celle adoptée par LECLERCQ et al. (1984). Le régime est 
distribué 2 fois par jour pour limiter le gaspillage. La quantité ingérée est calculée en soustrayant les refus à la fin de la période. Les fientes sont récoltées 2 fois par jour ; elles sont ensuite congelées puis lyophylisées et pesées après 24 heures d'exposition à la température ambiante. Elles sont ensuite broyées à l'aide d'un broyeur Dangoumeau pendant $4 \mathrm{~min}$ et stockées à l'abri de l'air.

\section{E. Méthodes analytiques}

La matière sèche est mesurée après passage à l'étuve à $105^{\circ} \mathrm{C}$ pendant 3 heures. Les protéines brutes sont estimées en multipliant par 6,25 l'azote obtenu par la méthode de Kjeldahl. Les matières azotées fécales non digestibles sont appréciées par la méthode de TERPSTRA \& de HART (1974). Elle consiste à précipiter les protéines contenues dans les fientes et supposées toutes d'origine fécale, par l'acétate de plomb à pH 4,7 en milieu acétique. Nous avons modifié la méthode en utilisant un bainmarie à agitation lente et en filtrant sous vide sur papier sans cendre de porosité élevée, en faisant en sorte que jusqu'à la fin du rinçage, les filtres ne soient jamais secs. Le coefficient de digestibilité azotée apparent $\left(\mathrm{CUD}_{\mathrm{az}}\right)$ est calculé suivant la formule :

$$
\mathrm{CUD}_{\mathrm{az}}=\frac{\text { protéines brutes ingérées }- \text { protéines brutes fécales } \times 1,18}{\text { protéines brutes ingérées }}
$$

Nous avons vérifié au préalable que la méthode de filtration n'influençait pas les résultats obtenus.

L'énergie est mesurée avec une bombe calorimétrique adiabatique Gallenkamp, modèle CB-110. Le calcul de l'énergie métabolisable non corrigée (EMnc) est obtenue suivant la formule :

$$
\mathrm{EMnc}=\mathrm{EB}_{\mathrm{i}}-\frac{\mathrm{F}}{\mathrm{A}} \mathrm{EB}_{\mathrm{f}}
$$

dans laquelle $\mathrm{EB}_{\mathrm{i}}$ est l'énergie brute en $\mathrm{Kcal} / \mathrm{kg}$ de matière sèche des aliments, $\mathrm{EB}_{\mathrm{f}}$ est l'énergie brute en $\mathrm{Kcal} / \mathrm{kg}$ de matière sèche des fientes, F/A est le rapport :

matière sèche totale des fientes excrétées

matière sèche totale des aliments ingérés

L'énergie métabolisable corrigée $(E M c)$ est obtenue en soustrayant de l'EMnc l'azote retenu, mesuré par le bilan azoté rapporté au kg d'aliment ingéré, multiplié par 8,22 selon la procédure adoptée par LecLERCQ et al. (1984).

\section{F. Méthodes statistiques}

Les calculs statistiques sont réalisés sur ordinateur en utilisant le programme Microstat-ITCF qui ne peut traiter que des effectifs égaux dans chaque lot. L'analyse de variance utilise le test de Newman et Keuls pour déterminer les valeurs significativement différentes. 


\section{Résultats et discussion}

Nous avons été amenés à éliminer un certain nombre d'animaux qui ont gaspillé des aliments au cours des périodes de bilan; nous en avons retenu 7 par lot. Nous étudierons successivement les valeurs obtenues pour l'énergie métabolisable, la digestibilité des protéines et le rapport F/A.

\section{A. Energie métabolisable}

Dans le tableau 3 sont rassemblés les résultats concernant l'EM corrigée pour le bilan azoté nul. En effet, l'analyse relative à l'EMnc aboutit aux mêmes conclusions car la valeur du terme correctif n'excède pas une dizaine de Kcalories, les bilans azotés les plus faibles correspondent à des ingérés plus petits. L'analyse de variance montre qu'il existe un effet significatif du génotype. Il porte sur un seul croisement,

\section{TABleaU 3}

Variation de l'énergie métabolisable corrigée pour le bilan azoté nul en fonction du génotype, de l'âge et du taux azoté des régimes.

Energy metabolizable variations with genotype, age and crude protein content.

\begin{tabular}{|c|c|c|c|c|c|c|}
\hline \multirow[b]{4}{*}{ Génotype } & \multicolumn{6}{|c|}{ Périodes } \\
\hline & \multicolumn{3}{|c|}{30 à 35 jours } & \multicolumn{3}{|c|}{55 à 60 jours } \\
\hline & \multicolumn{2}{|c|}{ Taux protéiques } & \multirow[t]{2}{*}{ Moyenne } & \multicolumn{2}{|c|}{ Taux protéiques } & \multirow[t]{2}{*}{ Moyenne } \\
\hline & 18 & 21 & & 14 & 18 & \\
\hline$J V 15$ & 3050 & 3119 & 3084 & 3119 & 3159 & 3139 \\
\hline$J \boldsymbol{A} 57 \ldots \ldots$ & 3075 & 3095 & 3085 & 3193 & 3069 & 3130 \\
\hline ISA Brown & 2947 & 2974 & 2960 & 3129 & 3093 & 3111 \\
\hline Fayoumi $\ldots \ldots$ & 3013 & 3087 & 3050 & 3072 & 3084 & 3078 \\
\hline
\end{tabular}

Analyse de variance.

Variance analysis.

\begin{tabular}{|c|c|c|c|}
\hline Source de variation & DDL & $\mathbf{F}$ & Probabilité (p. 100) \\
\hline 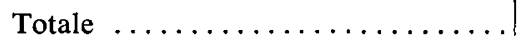 & 111 & & \\
\hline 1 Génotype ............... & 3 & 4,5 & 0,57 \\
\hline 2 Taux azoté $\ldots \ldots \ldots \ldots \ldots \ldots$ & 1 & 0,34 & 50 \\
\hline 3 Age $\ldots \ldots \ldots \ldots \ldots \ldots \ldots \ldots$ & 1 & 16,35 & 0,03 \\
\hline Interaction $12, \ldots \ldots \ldots \ldots \ldots \ldots$ & 3 & 2,04 & 11,2 \\
\hline$\ldots \ldots \ldots \ldots \ldots \ldots$ & 3 & 2,55 & 5,9 \\
\hline Interaction $23 \quad \ldots \ldots \ldots$ & 1 & 4,73 & 3,0 \\
\hline Interaction $123 \ldots \ldots \ldots \ldots \ldots \ldots$ & 3 & 0,52 & 50 \\
\hline Résiduelle $\ldots \ldots \ldots \ldots \ldots \ldots \ldots$ & 96 & & \\
\hline
\end{tabular}


l'Isa Brown, pour lequel l'EMnc est inférieure de $80 \mathrm{Kcalories}$ environ à celle obtenue pour les souches chair. Nous retrouvons ici d'une part l'absence d'effet du gène $d w$ sur l'énergie métabolisable observée par Guillaume (1969) et d'autre part une chute de l'EM correspondant à des croisements entre sujets issus de la race Rhode-Island Red. Comme la nature des régimes diffère entre les 2 périodes de mesure, nous avons également effectué une analyse de variance pour chacune d'elles. Il en résuite que les différences sont significatives au seuil de 5 p. 100 d'après la valeur de F durant la première période, mais ne le sont plus dans la deuxième, les écarts entre génotypes extrêmes devenant inférieurs à 40 Kcalories.

L'influence de l'âge est également significative, qu'elle soit appréciée à travers l'analyse de variance générale ou seulement pour le régime à 18 p. 100 de protéines brutes. L'augmentation moyenne de $70 \mathrm{Kcalories}$ entre la première et la deuxième période va dans le même sens que les résultats de ZeLENKA (1968) et Sorensen et al. (1983). Il est intéressant de noter que pour les 2 lots d'ISA Brown recevant le régime à 18 p. 100 de protéines brutes l'accroissement de l'EM est plus important que pour les autres génotypes. Peut-être faut-il y voir, comme dans les expériences de LESSIRE et al. (1982), une amélioration de la digestibilité du suif présent dans toutes nos rations. En ce sens les différences proviendraient principalement des variations de digestibilité des lipides en fonction du génotype.

\section{B. Digestibilité azotée}

Les valeurs des coefficients de digestibilité azotée sont rassemblées dans le tableau 4. Comme pour l'EM, l'influence du génotype se manifeste significativement. Ce sont également les sujets $I S A$ Brown qui possèdent les plus faibles CUD $_{\mathrm{az}}$. Ici également l'analyse de variance, période par période, montre que c'est seulement durant la première période que les différences sont hautement significatives; les écarts entre extrêmes dépassent 1,5 p. 100 , tandis qu'ils demeurent inférieurs à 0,6 p. 100 pour la deuxième période. Avec l'âge, la digestibilité diminue d'environ 0,7 p. 100 lorsque l'on prend en considération tous les lots ou seulement ceux qui reçoivent le régime à 18 p. 100 de protéines. Dans ce dernier cas, nous retrouvons les résultats d'Håkansson \& ERIKSSON (1974) et ceux de Fonolla et al. (1981). Cependant, il existe une interaction entre génotype et âge. Elle se traduit par une diminution de 1 à 2 p. 100 pour les souches chair et par une augmentation de 0,2 pour les $I S A$ Brown et de 0,8 p. 100 pour les Fayoumi. Cette diminution de la digestibilité en présence du gène $d w$ pourrait expliquer partiellement les besoins azotés plus élevés de ces oiseaux constatés par Gulllaume (1976). Des interactions existent entre taux azoté et génotype d'une part et âge d'autre part. Mais elles sont délicates à interpréter car la variation de la teneur en protéines des régimes résulte d'une profonde modification de la composition des rations principalement pour la séquence 18 et 14,3 p. 100 .

Cependant, il faut souligner les incertitudes dues à la méthodologie utilisée. N'ayant pas mesuré conjointement l'azote insoluble et l'acide urique, il est possible que la précipitation des protéines des fientes entraîne une fraction de produits urinaires liée à ces protéines. Nous remarquons toutefois que les variations d'azote insoluble observées par HÅKANSSON \& ERIKSSON (1974) portent essentiellement sur des périodes antérieures à celles où nous avons fait nos mesures. 


\section{TABleau 4}

Variation du coefficient de digestibilité azoté en fonction du génotype, de l'âge et du taux azoté.

Protein digestibility variation with genotype, age, and crude protein content.

\begin{tabular}{|c|c|c|c|c|c|c|}
\hline \multirow[b]{4}{*}{ Génotype } & \multicolumn{6}{|c|}{ Périodes } \\
\hline & \multicolumn{3}{|c|}{30 à 35 jours } & \multicolumn{3}{|c|}{55 à 60 jours } \\
\hline & \multicolumn{2}{|c|}{ Taux protéiques } & \multirow{2}{*}{ Moyenne } & \multicolumn{2}{|c|}{ Taux protéiques } & \multirow[t]{2}{*}{ Moyenne } \\
\hline & 18 & 21 & & 14 & 18 & \\
\hline JV 15 & 84,8 & 87,2 & 86,0 & 85,2 & 84,6 & 84,9 \\
\hline JA $57 \ldots \ldots$ & 86,3 & 87,0 & 86,6 & 86,7 & 82,7 & 84,7 \\
\hline ISA Brown .... & 84,0 & 84,7 & 84,3 & 85,5 & 84,1 & 84,8 \\
\hline Fayoumi ...... & 85,2 & 88,2 & 86,7 & 86,3 & 86,0 & 86,2 \\
\hline
\end{tabular}

Analyse de variance.

Variance analysis.

\begin{tabular}{|c|c|c|c|}
\hline Source de variation & DDL & $\mathbf{F}$ & Probabilité (p. 100) \\
\hline Totale & 111 & & \\
\hline 1 Génotype & 3 & 6,60 & 0,07 \\
\hline 2 Taux azoté $\ldots \ldots \ldots \ldots \ldots \ldots$ & 1 & 0,09 & 50 \\
\hline 3 Age $\ldots \ldots \ldots \ldots \ldots \ldots \ldots \ldots$ & 1 & 6,68 & 1,1 \\
\hline Interaction $12 \ldots \ldots \ldots \ldots \ldots \ldots \ldots$ & 3 & 5,00 & 0,3 \\
\hline Interaction $13 \ldots \ldots \ldots \ldots \ldots \ldots$ & 3 & 2,94 & 3,6 \\
\hline Interaction $23 \ldots \ldots \ldots \ldots \ldots \ldots$ & 1 & 29,34 & 0,01 \\
\hline Interaction $123 \ldots \ldots \ldots \ldots \ldots \ldots$ & 3 & 0,77 & 50 \\
\hline Résiduelle $\ldots \ldots \ldots \ldots \ldots \ldots \ldots$ & 96 & & \\
\hline
\end{tabular}

\section{Relation entre fientes et ingéré}

Les résultats correspondant au rapport $\mathrm{F} / \mathrm{A}$ sont rassemblés dans le tableau 5 . L'analyse de variance du rapport fiente/ingéré (F/A) montre que les Fayoumi excrètent significativement plus de fientes que les souches chair, que cette élimination diminue en fonction de l'âge, parallèlement à la diminution du taux azoté. La diminution la plus marquée correspond aux animaux les plus âgés recevant le régime à 14 p. 100 de protéines. Il existerait donc une diminution de l'excrétion urinaire puisque la digestibilité a tendance à augmenter chez ces poulets d'origine égyptienne. Mais ici encore les mêmes critiques méthodologiques peuvent être appliquées car nous n'avons pas séparé la part des excrétions endogènes ni analysé leur valeur. 
TABLEAU 5

$$
\begin{gathered}
\text { Variation du rapport } \frac{\text { matières sèches totales des fientes excrétées }}{\text { matières sèches totales de l'aliment ingéré }}\left(\frac{F}{A}\right) \\
\text { et du taux azoté. }
\end{gathered}
$$

\begin{tabular}{|c|c|c|c|c|c|c|}
\hline \multirow[b]{4}{*}{ Génotype } & \multicolumn{6}{|c|}{ Périodes } \\
\hline & \multicolumn{3}{|c|}{30 à 35 jours } & \multicolumn{3}{|c|}{55 à 60 jours } \\
\hline & \multicolumn{2}{|c|}{ Taux protéiques } & \multirow[t]{2}{*}{ Moyenne } & \multicolumn{2}{|c|}{ Taux protéiques } & \multirow[t]{2}{*}{ Moyenne } \\
\hline & 18 & 21 & & 14 & 18 & \\
\hline JV 15 & 0,28 & 0,28 & 0,28 & 0,22 & 0,27 & 0,25 \\
\hline$J A 57 \ldots \ldots$ & 0,27 & 0,29 & 0,28 & 0,21 & 0,28 & 0,25 \\
\hline ISA Brown & 0,30 & 0,29 & 0,30 & 0,23 & 0,27 & 0,25 \\
\hline Fayoumi ....... & 0,30 & 0,30 & 0,30 & 0,25 & 0,29 & 0,27 \\
\hline
\end{tabular}

$F / A$ ratio variations with genotype, age and crude protein content.

Analyse de variance.

\begin{tabular}{|c|c|c|c|}
\hline Source de variation & DDL & F & Probabilité (p. 100) \\
\hline Totale & 111 & & \\
\hline 1 Génotype & 3 & 3,71 & 1,4 \\
\hline 2 Taux azoté $\ldots \ldots \ldots \ldots \ldots \ldots$ & 1 & 6,98 & 0,001 \\
\hline 3 Age $\ldots \ldots \ldots \ldots \ldots \ldots \ldots \ldots$ & 1 & 56,58 & 0,001 \\
\hline Interaction $12 \ldots \ldots \ldots \ldots \ldots \ldots \ldots$ & 3 & 1,88 & 13,7 \\
\hline Interaction 13 & 3 & 0,56 & 50,0 \\
\hline Interaction $23 \ldots \ldots \ldots \ldots \ldots \ldots$ & 1 & 22,63 & 0,001 \\
\hline Interaction $123 \ldots \ldots \ldots \ldots \ldots \ldots$ & 3 & 0,22 & 50 \\
\hline Résiduelle $\ldots \ldots \ldots \ldots \ldots \ldots \ldots \ldots$ & 96 & & \\
\hline
\end{tabular}

Variance analysis.

\section{Corrélations entre les différentes mesures}

Nous avons aussi calculé les corrélations entre les différentes mesures. Elles figurent dans le tableau 6.

Il existe naturellement une corrélation étroite entre EMnc et EMc. Une corrélation positive élevée est également constatée entre les $2 \mathrm{EM}$ et le $\mathrm{CUD}_{\mathrm{az}}$ durant la première période ; elle est moins élevée durant la seconde. Il est possible qu'une sélection basée sur l'amélioration du rendement énergétique entraîne celle de la digestibilité azotée. 


\section{TABLEAU 6}

Corrélations entre les mesures pour les 2 périodes.

Correlations between measurements for the 2 periods

Période 30-35 jours

\begin{tabular}{l|c|c|c|c}
\hline \hline & F/A ${ }^{(*)}$ & EMnc & EMc & CUDaz \\
\hline F/A $\ldots \ldots \ldots \ldots \ldots \ldots$ & 1 & & & \\
EMnc $\ldots \ldots \ldots \ldots \ldots \ldots$ & $-0,857$ & 1 & & \\
EMc $\ldots \ldots \ldots \ldots \ldots \ldots$ & $-0,857$ & 0,999 & 1 & \\
CUDaz $\ldots \ldots \ldots \ldots \ldots$ & $-0,541$ & 0,819 & 0,817 & 1 \\
\hline
\end{tabular}

Période 55-60 jours

\begin{tabular}{l|c|c|c|c}
\hline & F/A & EMnc & EMc & CUDaz \\
\hline F/A $\ldots \ldots \ldots \ldots \ldots \ldots$ & 1 & & \\
EMnc $\ldots \ldots \ldots \ldots \ldots$ & $-0,709$ & 1 & & \\
EMc $\ldots \ldots \ldots \ldots \ldots$ & $-0,709$ & 0,999 & 1 & \\
CUDaz $\ldots \ldots \ldots \ldots$ & $-0,605$ & 0,580 & 0,587 & 1 \\
\hline
\end{tabular}

Toutes les valeurs sont hautement significatives (probabilité de 0,1 p. 100).

(*) Voir les définitions dans le paragraphe II.E.

Plus intéressante est la corrélation entre le rapport F/A et EM. Mais sa signification demande d'autres études pour comprendre l'évolution avec le temps de l'excrétion azotée d'origine digestive et urinaire.

\section{Conclusion}

Cette étude confirme l'existence de différences entre génotypes portant sur la valeur de l'énergie métabolisable et sur la digestibilité azotée. Elles sont cependant de trop faible amplitude pour intéresser le sélectionneur. En revanche, leur existence pourrait être prise en compte lors d'un choix de lignées destinées à être croisées entre elles, en particulier lorsqu'il s'agit de sujets issus de race Rhode-Island Red. Ces variations fluctuent avec l'âge, probablement à la suite de l'amélioration de la digestibilité des acides gras saturés. Cette hypothèse mériterait d'être confirmée par d'autres expériences.

D'un autre côté, les variations concomitantes de l'EM et du rapport F/A en fonction du génotype et de l'âge mériteraient d'être approfondies pour mieux comprendre l'évolution de l'excrétion fécale et urinaire en fonction du génotype et de l'âge des poulets.

Reçu le 23 octobre 1985. Accepté le 17 décembre 1985. 


\section{Références bibliographiques}

ANONYME, 1984. Alimentation des animaux monogastriques : porcs, lapins, volailles. 282 pp., I.N.R.A., Paris.

BEgIN J.J., 1967. The relation of breed and sex of chickens to the utilization of energy. Poult. Sci., 46, 379-383.

BEGIN J.J., 1969. The effect of diet and breed of chicken on the metabolic efficiency of nitrogen and energy utilization. Poult. Sci., 48, 48-54.

Fonolla J., Prieto C., Sanz R., 1981. Influence of age on the nutrient utilization of diets for broilers. Anim. Feed Sci. Technol., 6, 405-411.

FosTeR W.H., 1968. Variation between and within birds in the estimation of metabolizable energy content of diets for laying hens. J. Agric. Sci. Camb., 71, 153-159.

Guillaume J., 1969. Conséquences de l'introduction du gène de nanisme dw sur l'utilisation alimentaire chez le poussin femelle. Ann. Biol. Anim. Bioch. Biophys., 9, 369-378.

Guillaume J., 1976. The dwarfism gene dw : its effects on anatomy, physiology, nutrition, management. Its application in poultry industry. World's Poult. Sci., 32, 285-304.

Häansson J., ERIKSSON S., 1974. Digestibility, nitrogen retention and consumption of metabolizable energy by chickens on feeds of low and high concentration. Swedish J. Agric. Res., 4, 195-207.

LeCLerce B., 1980. La valeur énergétique des matières premières : influence de l'âge, du sexe et du type génétique et comparaison entre espèces avicoles. In : Matières premières et alimentation des volailles, 65-78, I.N.R.A., Nouzilly.

Leclerce B., Prevotel B., Carré B., 1984. Equation de prédiction de la valeur énergétique des aliments composés destinés aux volailles : étude expérimentale. 69 pp., I.N.R.A., Nouzilly.

Lessire M., Leclerce B., Connan L., 1982. Metabolisable energy value of fats in chicks and adult cockerels. Anim. Feed. Sci. Technol., 7, 365-374.

Milller W.S., 1974. The determination of metabolizable energy. In : MoRRIS T.R., Freeman B.M. (ed.), Energy requirement of poultry, 91-112. Brit. Poult. Sci. Ltd, Edinburgh.

Proudman J.A., Mellen W.J., Anderson D.L., 1970. Utilization of feed in fast- and slow-growing lines of chickens. Poult. Sci., 49, 961-972.

Sibbald I.R., Slinger S.J., Ashton G.C., 1961. Factors affecting the metabolizable energy content of poultry feeds. 2 . Variability in the ME values attributed to samples of tallow, and undegummed soybean oil. Poult. Sci., 40, 303-308.

Sibbald I.R., Slinger J.J., 1963. The effects of breed, sex and arsenical and nutrient density on the utilization of dietary energy. Poult. Sci., 42, 1325-1332.

Slinger S.J., Sibbald I.R., Pepper W.F., 1964. The relative abilities of two breed of chickens and two varieties of turkeys to metabolize dietary energy and dietary nitrogen. Poult. Sci., 43, 329-333.

Serensen P., Chwalibog A., Eggum B.O., 1983. Protein and energy metabolism in two lines of chickens selected for growth on high or low protein diets. Brit. Poult. Sci., 24, 237-250.

Terpstra K., de Hart N., 1974. The estimation of urinary nitrogen and faecal nitrogen in poultry excreta. Z. Tierphysiol. Tierernährg u. Futtermittelkde, 32, 306-320.

ZELENKA J., 1968. Influence of the age of chicken on the metabolizable enery values of poultry diets. Brit. Poult. Sci., 9, 135-142. 